\title{
A new mechanical band pass filter design for energy harvesting
}

\author{
Alireza Keshmiri, Nan Wu \\ Department of Mechanical Engineering \\ University of Manitoba \\ Winnipeg, Canada \\ keshmira@myumanitoba.ca, Nan.Wu@umanitoba.ca
}

\begin{abstract}
This work presents an analytical methodology for development of a mechanical band pass filter for energy scavenging. The model is based on Adomian decomposition method to derive the dynamic response of the non-uniform smart structure with strain rate damping to external environmental excitations and efficiently harvest the subsequent vibrational energy. The steady state response of the nonlinearly tapered cantilever beam subjected to a harmonic base motion is obtained and electromechanical outputs in open circuit condition are analytically derived. In other to design a band pass filter, an array of cantilever beams with nonlinear geometry and surface bonded piezoelectric layers are considered. Therefore, the filter can choose one harvester to resonate at one particular ambient vibration frequency and reach the maximum electromechanical output. Compared with uniform beam design, it is also demonstrated that the proposed non-uniform configuration can generate higher electrical output over a wide frequency range.
\end{abstract}

Keywords - Band pass filter; Piezoelectric energy harvesting; Non-uniform structure; Adomian decomposition method.

\section{INTRODUCTION}

Energy harvesting from ambient energy sources has become a dynamic research field and received so much attention in recent years because of huge number of applications. With decreasing electrical power requirements in wireless electronics and increasing utilization of sensors in different structures, energy harvesting to power sensors at the location without batteries limitations is appealing for various engineering automation and monitoring systems. Therefore, energy harvesters are designed to convert the environment energies into usable power form to supply the small wireless sensor systems throughout the sensor lifetime.

Alternatively, energy scavenging based on vibration is becoming more attractive. Conversion of mechanical vibration energy into electrical energy can be achieved through three conversion mechanisms: electrostatic, electromagnetic, and piezoelectric [1]. The small size, custom shape fabrication ability and high power conversion potential are the key and fundamental advantages of piezoelectric approach for vibration energy harvesting [2]. Even a small external excitation leads to considerable amount of generated voltage without requiring any low-efficient initiation mechanical mechanism [3].

The most common energy harvester design is the cantilever beam with surface bonded piezoelectric layers transversely vibrating because of the base excitation. Although it is not essentially the best design in terms of output energy, it is wellknown because of its simple and cost-efficient configuration. Furthermore, most of piezoelectric energy harvesters work on the resonance mechanism to raise the strain distribution in the piezoelectric layer and eventually increase the output electrical power. Due to the small size factor, resonance frequency of the harvester increases and makes it undesirable for low frequency vibrations. The common response to this problem is adding a proof mass at the free end $[4,5]$. But, this optimal resonance condition is not given in most of the environmental cases with variable vibration conditions. Given this, having a single harvester design capable of operating over a range of vibration frequencies would be desirable. Additionally, refining the geometry of the scavenger is itself a major improvement area. A trapezoidal profile can distribute the strain evenly such that maximum stain is obtained at every point in the piezoelectric energy harvester. A harvester with trapezoidal geometry can generate more than twice energy than a harvester with rectangular shape [6].

One of the biggest disadvantages of common vibration energy harvesters is the very limited practical bandwidths over which energy can be scavenged. In order to increase the frequency range of vibration energy harvesters, two approaches of tuning the resonant frequency and widening the bandwidth are suggested in the literature [7]. Based on the widen bandwidth methodology, the operational frequency range of the vibration energy generator can be extended by using an array of harvesters with different natural frequencies. In other words, the piezoelectric energy harvester should have appropriate bandwidth in the range of peak-power frequencies of the ambient vibrations. This frequency range can be easily measured in different environments. Such a device with this particular characteristic can be called a piezoelectric band pass filter [5].

The work presented in this research endeavors to challenge previous energy harvesting designs and fabricate a novel and advanced energy harvester system that possesses enough bandwidth in the range of ambient vibration frequencies. The model developed in this paper has included nonlinear geometry 
rather than a simple uniform pattern. The dynamic steady state solution of the harmonic base excitation problem for lateral vibration of the non-uniform smart structure with strain rate damping is presented and electromechanical outputs of the piezoelectric patches in open circuit condition are analytically derived. The mathematical process is based on Adomian decomposition method (ADM) and the mechanical model is applicable to any slender beam which allows neglecting shear deformation and rotatory inertia effects. Finally, it is demonstrated that such an arrangement of non-uniform cantilever piezoelectric coupled beams can be made into a band pass filter when the dimensions are selected properly.

\section{THEORETICAL MODEL}

The proposed bimorph piezoelectric energy harvester configuration consists of a nonlinearly tapered cantilever beam with piezoelectric patches bonded on the top and bottom surfaces, Fig. 1. The geometry of the structure is described by $L$ length of the beam, $2 b_{0}$ width of the beam at the fixed end, $2 h_{0}$ thickness of the beam at the fixed end, $L_{p}$ the piezoelectric patch length and $h_{p}$ thickness of the piezoelectric layer. The energy harvesting efficiency of the piezoelectric coupled cantilever beam can be investigated by setting the mechanical vibration input for the system in the form of $f(t)=Y e^{j \varphi t}$ where $Y$ is the amplitude of the base displacement, $\varphi$ is the angular frequency of the vibration and $j$ is the unit imaginary number. The differential equation that describes the forced lateral vibration of a smart beam with non-uniform geometry and structural strain rate damping can be expressed by,

$$
\rho A(x) \frac{\partial^{2} w(x, t)}{\partial t^{2}}+\frac{\partial^{2}}{\partial x^{2}}\left[E I(x) \frac{\partial^{2} w(x, t)}{\partial x^{2}}+C_{s} I(x) \frac{\partial^{3} w(x, t)}{\partial x^{2} \partial t}\right]=-\rho A(x) \phi^{2} Y e^{j \omega x}
$$

where $x$ is position variable along length of the beam, $t$ is time variable, $\rho$ is the equivalent mass density, $A(x)$ is crosssectional area, $w(x, t)$ is the deflection function, $E$ is the equivalent elasticity modulus, $I(x)$ is the second moment of area and $C_{s}$ is the strain-rate damping coefficient. Moreover, boundary conditions for the non-uniform cantilever beam are defined as,

$$
\begin{aligned}
& {[w(x, t)]_{x=0}=0,\left[\frac{\partial w(x, t)}{\partial x}\right]_{x=0}=0,} \\
& {\left[E I(x) \frac{\partial^{2} w(x, t)}{\partial x^{2}}+C_{s} I(x) \frac{\partial^{3} w(x, t)}{\partial x^{2} \partial t}\right]_{x=L}=0,} \\
& {\left[\frac{\partial}{\partial x}\left(E I(x) \frac{\partial^{2} w(x, t)}{\partial x^{2}}+C_{s} I(x) \frac{\partial^{3} w(x, t)}{\partial x^{2} \partial t}\right)\right]_{x=L}=0 .}
\end{aligned}
$$

Using the normal mode approach, the solution of Eq. (1) is assumed to be a linear combination of the normal modes of the beam denoted by a convergent series as,

$$
w(x, t)=\sum_{i=1}^{\infty} X_{i}(x) T_{i}(t),
$$

where $X_{i}(x)$ are mass normalized modes and $T_{i}(t)$ are modal participation coefficients for the $i$ th vibration mode. Since the system is proportionally damped, eigenfunctions represented by $X_{i}(x)$ are normal mode eigenfunctions of the corresponding undamped free vibration problem $[8,9]$.

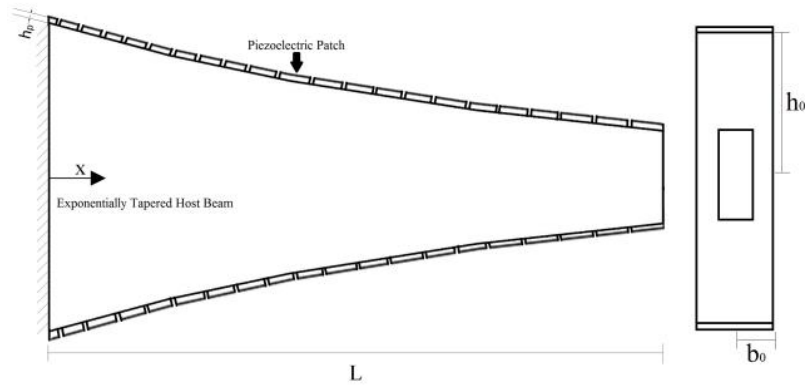

Figure 1. A nonlinearly tapered cantilever beam with piezoelectric patches mounted on its surface, side and front views.

Consequently, normal modes can be calculated by solving,

$$
E I(x) \frac{d^{4} X_{i}(x)}{d x^{4}}+2 E \frac{d I(x)}{d x} \frac{d^{3} X_{i}(x)}{d x^{3}}+E \frac{d^{2} I(x)}{d x^{2}} \frac{d^{2} X_{i}(x)}{d x^{2}}-\omega_{i}^{2} \rho A(x) X_{i}(x)=0
$$

where $\omega_{\mathrm{i}}^{2}$ are the undamped natural frequencies of the structure.

In order to solve the Eq. (4), ADM is used. The solution obtained by ADM is an infinite series; an $n$-term approximation usually serves as a practical solution. Since the methodology itself is not the main focus of this research and it is perfectly addressed in [10,11], more details will not be discussed here. Hence, by applying ADM, solution of Eq. (4) which is the mode shape function in the recurrence format can be expressed as,

$$
X_{i}(x)=\sum_{k=0}^{\infty} X_{k_{i}}(x)=X_{0}-L_{x}^{-1}\left\{\begin{array}{l}
2 \frac{\frac{d I(x)}{d x}}{I(x)} \sum_{k=0}^{\infty} \frac{d^{3} X_{k_{i}}(x)}{d x^{3}} \\
+\frac{d^{2} I(x)}{d x^{2}} \sum_{k=0}^{\infty} \frac{d^{2} X_{k_{i}}(x)}{d x^{2}} \\
-\omega_{i}^{2} \frac{\rho A(x)}{E I(x)} \sum_{k=0}^{\infty} X_{k_{i}}(x)
\end{array}\right\}
$$

where the inverse operator $L_{x}^{-1}$ is a four fold integration with respect to $\mathrm{x}$ Additionally, $X_{0}=C_{1}+C_{2} x+C_{3} x^{2} / 2 !+C_{4} x^{3} / 3$ ! and $C_{1}$ to $C_{4}$ are constants which can be evaluated from the system boundary conditions. Thus, all $X_{k}$ components are evaluated and the mode shape function is obtained. As many terms as required to obtain a more accurate shape function can be considered.

Clearly, normal mode eigenfunctions satisfy the orthogonality conditions,

$$
\begin{aligned}
& \int_{x=0}^{L} \rho A(x) X_{m}(x) X_{n}(x) d x=\left\{\begin{array}{l}
1 \rightarrow m=n \\
0 \rightarrow m \neq n
\end{array}\right. \\
& \int_{x=0}^{L} \frac{d^{2}}{d x^{2}}\left[E I(x) \frac{d^{2} X_{m}(x)}{d x^{2}}\right] X_{n}(x) d x=\left\{\begin{array}{c}
\omega_{m}^{2} \rightarrow m=n \\
0 \rightarrow m \neq n
\end{array}\right.
\end{aligned}
$$

Therefore, the modal participation coefficients $T_{i}(t)$ are the solution of the ordinary differential equation, 


$$
\begin{aligned}
& \frac{d^{2} T_{i}(t)}{d t^{2}}+2 \xi_{i} \omega_{i} \frac{d T_{i}(t)}{d t}+\omega_{i}^{2} T_{i}(t)= \\
& Q_{i}(t)=\rho \phi^{2} Y\left[\int_{0}^{L} X_{i}(x) A(x) d x\right] e^{j \phi t},
\end{aligned}
$$

where $2 \xi_{\mathrm{i}} \omega_{\mathrm{i}}=C_{s} \omega_{\mathrm{i}}^{2} / E$ and $\xi_{\mathrm{i}}$ is the structural strain-rate damping ratio of $i$ th vibration mode. Finally, the modal response can be obtained by Duhamel integral,

$$
T_{i}(t)=\frac{1}{\omega_{d i}} \int_{0}^{t} Q_{i}(\tau) e^{-\xi_{i j}(t-\tau)} \sin \omega_{d i}(t-\tau) d \tau,
$$

where $\omega_{\mathrm{di}}=\omega_{\mathrm{i}}\left[1-\xi_{\mathrm{i}}^{2}\right]^{0.5}$ is the $i$ th mode damped natural frequency. However, the steady state solution of Eq. (7) can be expressed as,

$$
T_{i}(t)=\frac{\rho \phi^{2} Y \int_{0}^{L} X_{i}(x) A(x) d x}{\omega_{i}^{2}-\phi^{2}+j 2 \xi_{i} \omega_{i} \phi} e^{j \phi t} .
$$

As a result, normal mode eigenfunctions given by Eq. (5) and modal participation coefficients obtained by Eq. (9) can be used in Eq. (3) to represent the dynamic steady state response of the smart structure relative to its base as,

$$
w(x, t)=\sum_{i=1}^{\infty} X_{i}(x) T_{i}(t)=\sum_{i=1}^{\infty} X_{i}(x) \frac{\rho \phi^{2} Y \int_{0}^{L} X_{i}(x) A(x) d x}{\omega_{i}^{2}-\phi^{2}+j 2 \xi_{i} \omega_{i} \phi} e^{j \phi t} .
$$

In order to obtain the electrical outputs of the piezoelectric energy harvester, the following piezoelectric constitutive equation is considered,

$$
D_{3}(x, t)=d_{31} \sigma_{1}(x, t)+e_{33}^{\sigma} E_{3}(t),
$$

where $D_{3}$ is the electrical displacement, $d_{31}$ (Coulomb/ $\mathrm{N}$ or $\mathrm{m} / \mathrm{V}$ ) is the piezoelectric coefficient with polarization in direction 3 due to the external stress in direction $1, \sigma_{l}$ is the axial stress along 1 -axis, $e_{33^{\sigma}}$ is the permittivity at constant stress and $E_{3}$ is the electrical field along 3-axis [12]. It should be noted that 1,2 and 3 directions are aligned with $x, y$ and $z$ axes, respectively.

By replacing the axial stress $\sigma_{1}$ with bending strain $\varepsilon_{1}$ and knowing that there is no applied electric field on the piezoelectric patch $E_{3}(t)=0$, Eq. (11) can be rewritten as,

$$
D_{3}(x, t)=d_{31} E_{p} \varepsilon_{1}(x, t),
$$

where $E_{p}$ is the piezoelectric elasticity modulus and $V(t)$ is the electric voltage over the piezoelectric area. Furthermore, the longitudinal bending strain $\varepsilon_{l}(x, t)$ in the structure can be calculated as,

$$
\varepsilon_{1}(x, t)=-h_{c} \frac{\partial^{2} w(x, t)}{\partial x^{2}}=-\left(h(x)+\frac{h_{p}}{2}\right) \frac{\partial^{2} w(x, t)}{\partial x^{2}},
$$

where $h_{c}$ is the distance between center of the piezoelectric layer and the beam's neutral axis and $\delta^{2} w(x, t) / \delta x^{2}$ is the curvature of the structure. Consequently, the electric displacement becomes,

$$
D_{3}(x, t)=-d_{31} E_{p}\left(h(x)+\frac{h_{p}}{2}\right) \frac{\partial^{2} w(x, t)}{\partial x^{2}} .
$$

The electric displacement $D_{3}(x, t)$ is related to the output electric charge $q(t)$ by integration over the electrode area. Since the vibration mode of the energy harvester is not restricted to the first mode, segmented piezoelectric patches and electrodes in order to harvest the maximum output charge are utilized. This is due to the fact that using continuous electrodes results in phase difference in the strain distribution which leads to charge cancellation [8].

$$
q(t)=-\sum_{k=1}^{N_{p}}\left\{\int_{x=(k-1) L_{p}}^{k L_{p}} d_{31} E_{p} b(x)\left(h(x)+\frac{h_{p}}{2}\right) \frac{\partial^{2} w(x, t)}{\partial x^{2}} d x\right\},
$$

where $N_{p}$ is the total number of piezoelectric patches mounted on the surface of the beam and obviously $N_{p} L_{p}=L$.

The generated voltage $v(t)$ can be calculated by dividing the produced electric charge to the capacitance as,

$$
v(t)=\frac{q(t)}{C_{p}}=-\frac{d_{31} E_{p}}{C_{p}} \sum_{k=1}^{N_{p}}\left\{\int_{(k-1) L_{p}}^{k L_{p}} b(x)\left(h(x)+\frac{h_{p}}{2}\right) \frac{\partial^{2} w(x, t)}{\partial x^{2}} d x\right\},
$$
layer.

where $C_{p}=e_{33}{ }^{\varepsilon} L b_{0} / h_{p}$ is the capacitance of the piezoelectric

The goal of this research is to estimate the open circuit voltage across the piezoelectric layers. So the backward coupling in the relations is ignored. Finally, steady state voltage can be obtained by using Eq. (10) in Eq. (16) as,

$$
v(t)=-\frac{d_{31} E_{p} \rho \phi^{2} Y}{C_{p}} \sum_{i=1}^{\infty}\left\{\sum_{k=1}^{N_{p}}\left[\int_{(x-1) L_{p}}^{k_{p}} b(x)\left(h(x)+\frac{h_{p}}{2}\right) \frac{d^{2} X_{i}(x)}{d x^{2}} d x\right] \frac{\int_{0}^{L} X_{i}(x) A(x) d x}{\omega_{i}^{2}-\phi^{2}+j 2 \xi_{i} \omega_{i} \phi}\right\} e^{j i t} .
$$

In order to provide a more general form of steady state response and not considering specific $Y$ and $\varphi$ values, frequency response functions (FRFs) of the electromechanical outputs are obtained and presented. Firstly, the voltage FRF is defined as the ratio of the steady state voltage output to the base acceleration. Hence, voltage FRF is described as,

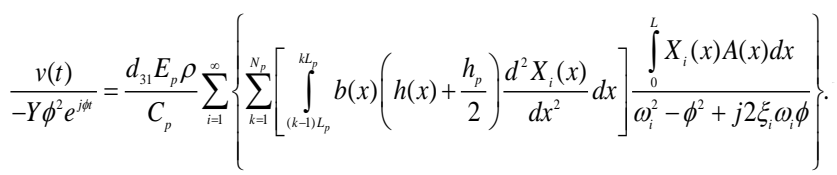

Secondly, the relative tip motion FRF is presented as ratio of the beam vibration amplitude at the tip to the amplitude of the base excitation. It is worth mentioning that the presented model is not limited to the motion at the tip and can be applied to any point along the beam. By using Eq. (10), tip motion FRF is defined as,

$$
\frac{w(L, t)}{Y e^{j \phi t}}=\rho \phi^{2} \sum_{i=1}^{\infty} X_{i}(L) \frac{\int_{0}^{L} X_{i}(x) A(x) d x}{\omega_{i}^{2}-\phi^{2}+j 2 \xi_{i} \omega_{i} \phi} .
$$

When the excitation frequency is equal to the natural frequency of the $i$ th mode $\left(\varphi=\omega_{i}\right)$, all the terms in the FRF 
relations other than the mode of interest can be ignored. As a result, the amplitude of voltage output and tip motion FRFs are rewritten as,

$$
\begin{gathered}
\left\|\frac{v(t)}{-Y \phi^{2} e^{j \phi t}}\right\|=\frac{d_{31} E_{p} \rho}{2 \xi_{i} \omega_{i}^{2} C_{p}}\left\{\sum_{k=1}^{N_{p}}\left[\int_{(k-1) L_{p}}^{k L_{p}} b(x)\left(h(x)+\frac{h_{p}}{2}\right) \frac{d^{2} X_{i}(x)}{d x^{2}} d x\right]\right\}\left[\int_{0}^{L} X_{i}(x) A(x) d x\right] . \\
\left\|\frac{w(L, t)}{Y e^{j \phi t}}\right\|=\frac{\rho \phi^{2}}{2 \xi_{i}} X_{i}(L) \int_{0}^{L} X_{i}(x) A(x) d x .
\end{gathered}
$$

\section{MECHANICAL BAND PASS FILTER}

In this section, the probability of designing a mechanical band pass filter from an array of non-uniform beams is investigated. The material properties and electromechanical coefficients are listed in Table 1. The excitation of the piezoelectric energy harvester is due to the harmonic base translation and the steady state dynamic behavior of the system is of interest. Furthermore, structural strain-rate damping ratios for the first and second vibration modes are assumed to be $\xi_{1}=\xi_{2}=0.03$. Finally, two steady state electromechanical FRFs, voltage output and beam tip motion, are examined.

TABLE I. GEOMETRY AND MATERIAL PROPERTIES OF THE PIEZOELECTRIC ENERGY HARVESTER.

\begin{tabular}{|c|c|c|c|c|}
\hline \multicolumn{5}{|c|}{ Host beam } \\
\hline $\boldsymbol{L}(\boldsymbol{m})$ & $\boldsymbol{b}_{\boldsymbol{0}}(\boldsymbol{m})$ & $\boldsymbol{h}_{\boldsymbol{0}}(\boldsymbol{m})$ & $\boldsymbol{E}(\boldsymbol{G P a})$ & $\rho\left(\mathbf{k g} / \boldsymbol{m}^{3}\right)$ \\
\hline 0.3 & 0.05 & 0.005 & 210 & 7800 \\
\hline \multicolumn{5}{|c|}{ PZT } \\
\hline $\boldsymbol{h}_{p}(\boldsymbol{m})$ & $\boldsymbol{E}_{p}(\mathbf{G P a})$ & $\rho\left(\mathbf{k g} / \boldsymbol{m}^{3}\right)$ & $\boldsymbol{d}_{\mathbf{3 1}}(\mathbf{p m} / \mathbf{V})$ & $\mathbf{e}_{33^{\varepsilon}}(\mathbf{n F} / \mathbf{m})$ \\
\hline 0.0004 & 66 & 7800 & -190 & 15.93 \\
\hline
\end{tabular}

In order to design a mechanical band pass filter, an array of non-uniform beams is considered. The idea of an ensemble of beam-mass system working as a band pass filter is borrowed from [5] and further developed in here. The system consists of several nonlinearly tapered piezoelectric coupled cantilever beams, each of which has different geometrical taper ratio and hence different resonance frequencies. Consequently, the assembled system has a wide operational frequency range and the filter can automatically choose one harvester to resonate at one particular ambient vibration frequency. When the natural frequency of the energy harvester matches the peak power frequency of the source, the output power is the most efficient. Additionally, to partially overcome the stability and size drawbacks, all the harvesters in the system have similar length, volume and mass. As a result, the entire system can work as a mechanical band pass filter depending on geometrical dimensions and taper ratios. Although the non-uniformity function in the presented model is not constrained to any specific type of function and is a general definition, here an exponential variation function $g(x)=g_{0} e^{-m x / L}$ is considered where $g_{0}$ is the initial value, $m$ is the taper ratio and $0 \leq x \leq L$.

Fig. 2 represents the nonlinear geometry effect on the electric voltage output in frequency domain. As can be seen, the amplitude of the voltage output drastically increases when the excitation frequency is close to the fundamental natural frequency of the harvester. It is worthwhile to mention that for every excitation frequency, maximum electrical output is gained when the piezoelectric energy harvester is working in open circuit condition [13]. As shown, higher nonlinearity in geometry of the harvester results in substantial increase of the voltage amplitude. For instance, by increasing the geometry taper ratio from 0 to 0.8 , the voltage output for the first two vibration modes show up to 15.46 times higher outputs, Fig. 2. Therefore, a system comprising only five non-uniform energy harvesters with different taper ratios and same volume/mass can function like a band pass filter over a wide frequency band $\left[\omega_{\min }-\omega_{\max }\right]=[290-550] \mathrm{rad} / \mathrm{s} \approx[45-90] \mathrm{Hz}$. Besides, the proposed non-uniform design substantially increases the voltage output of the system compared to the uniform one [5].

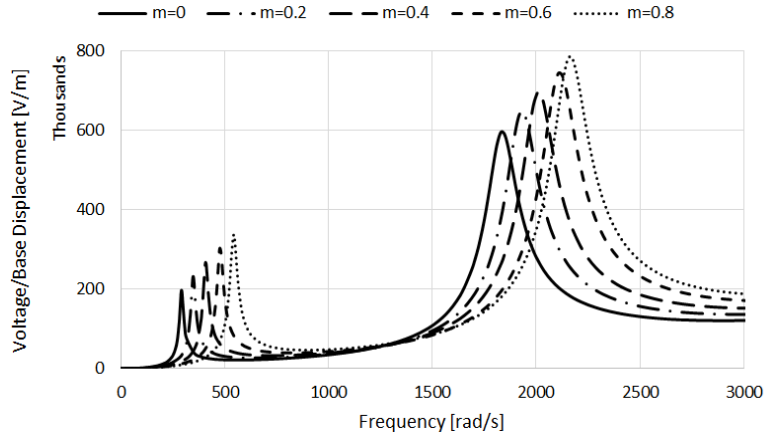

Figure 2. Voltage output FRF for an array of non-uniform piezoelectric energy harvesters with five different geometrical taper ratios.

Finally, the tip displacement in frequency domain for various nonlinear geometries is shown in Fig. 3. Likewise, increasing the nonlinearity in the geometry of the harvesters leads to higher natural frequencies and tip displacements. As mentioned earlier, trapezoidal profile can distribute the strain evenly such that maximum strain is obtained at every point in the piezoelectric energy harvester.

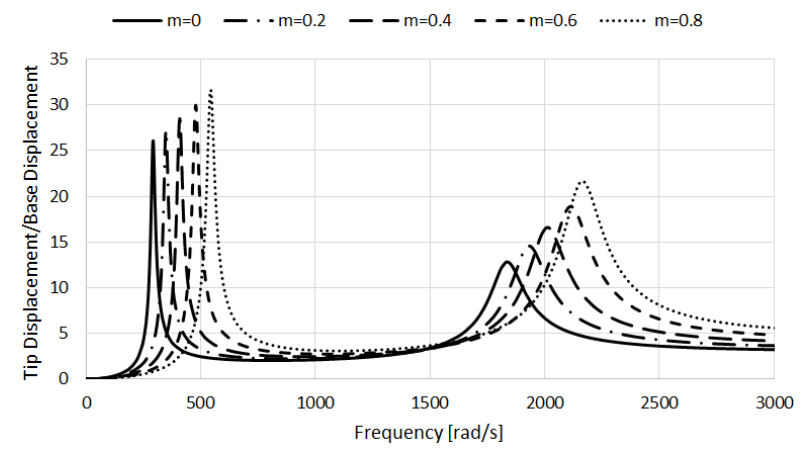

Figure 3. Beam tip motion FRF for an array of non-uniform piezoelectric energy harvesters with five different geometrical taper ratios.

\section{CONCLUSION}

In this paper, an analytic electromechanical model for a nonlinearly tapered cantilever piezoelectric energy harvester is presented. The non-uniform piezoelectric harvester is assumed to be excited by harmonic base motion in the transverse direction. By using ADM, the steady state dynamic behavior of the smart structure with non-uniform geometry and structural strain-rate damping is obtained and closed-form expressions for the electromechanical parameters are derived. Analytically 
obtained equations are used to determine the electric charge, voltage output and tip motion FRFs to be able to see the frequency response of the non-uniform harvesters. The electromechanical FRFs are plotted against frequency in open circuit condition which leads to the maximum output. Finally, a mechanical band pass filter including an array of nonlinearly tapered piezoelectric bimorph cantilever beams, each of which has different geometrical taper ratio and hence different resonance frequencies is designed and presented.

In summary, a new approach to increase the frequency range and electrical outputs of vibration energy harvesters by widening the bandwidth of the generator and introducing nonlinear structural geometry design is developed and presented. This is achieved through employing an array of nonuniform harvesters with different resonance frequencies. The new nonlinear geometry design has demonstrated to be promising to considerably increase the electromechanical outputs of the band pass filter in a wide frequency domain. This research challenges previous energy harvesting designs and formulates an innovative harvester configuration which functions optimally and efficiently on extensive range of ambient vibration sources.

\section{ACKNOWLEDGMENT}

This research was undertaken, in part, thanks to funding supports from University of Manitoba, Research Manitoba and Natural Sciences and Engineering Research Council of Canada (NSERC).

\section{REFERENCES}

[1] S. Roundy, P. K. Wright and J. Rabaey, "A study of low level vibrations as a power source for wireless sensor nodes," Computer Communications, vol. 26, no. 11, pp. 1131-1144, 2003.

[2] N. G. Elvin, A. A. Elvin and M. Spector, "A self-powered mechanical strain energy sensor," Smart Materials and Structures, vol. 10, pp. 293299, 2001.

[3] T. Hehn and Y. Manoli, CMOS Circuits for piezoelectric energy harvesters, Netherlands: Springer, 2015.

[4] A. Erturk and D. J. Inman, "Mechanical considerations for modeling of vibration-based energy harvesters," in Proceedings of the ASME IDETC 21st Biennial Conference on Mechanical Vibration and Noise, Las Vegas, NV, 2007.

[5] S. Shahruz, "Design of mechanical band-pass filters for energy scavenging," Journal of Sound and Vibration, vol. 292, pp. 987-998, 2006.

[6] S. Roundy, E. S. Leland, J. Baker, E. Carleton, E. Reilly, E. Lai, B. Otis, J. M. Rabaey and P. K. Wright, "Improving power output for vibrationbased energy scanvengers," Energy Harvesting and Conservation, vol. 4, no. 1, pp. 28-36, 2005.

[7] D. Zhu, M. J. Tudor and S. P. Beeby, "Strategies for increasing the operating frequency range of vibration energy harvesters: a review," Measurement Science and Technology, vol. 21, pp. 1-29, 2010.

[8] A. Erturk and D. J. Inman, "On mechanical modeling of cantilevered piezoelectric vibration energy harvesters," Journal of Intelligent Material Systems and Structures, vol. 19, no. 11, pp. 1311-1325, 2008.

[9] T. K. Caughey and M. E. O'Kelly, "Classical normal modes in damped linear dynamic systems," ASME Journal of Applied Mechanics, vol. 32, pp. 583-588, 1965.

[10] G. Adomian, "A Review of the Decomposition Method in Applied Mathematics," Journal of Mathematical Analysis and Applications, vol. 135 , pp. 501-544, 1988.
[11] A. Keshmiri, N. Wu and Q. Wang, "Free vibration analysis of a nonlinearly tapered cone beam by Adomian decomposition method," International Journal of Structural Stability and Dynamics, 2018.

[12] IEEE Standard on Piezoelectricity, New York: IEEE, 1987.

[13] A. Erturk and D. J. Inman, "A distributed parameter electromechanical model for cantlievered piezoelectric energy harvesters," Journal of Vibration and Acoustics, vol. 130, pp. 1-14, 2008. 